Conclusion HCV core $_{35-44}$ inhibits NK cell activity via two distinct mechanisms, directly via KIR2DL2/3, and synergistically via the CD94:NKG2A receptor. This synergistic interaction at CD94:NKG2A represents a novel mechanism for inhibiting NK cells. It demonstrates the sensitivity of NK cells to small changes in the peptide content of HLA class I, and is thus potentially a sensitive mechanism for viral escape from the host innate immune response.

\section{P92 CD161 $+\gamma-\delta$ T CELLS: DEFINING THEIR ROLE IN PATIENTS WITH AND WITHOUT CHRONIC HEPATITIS C}

doi:10.1136/gutjnl-2011-300857a.92

${ }^{1} \mathrm{~N}$ Rajoriya, ${ }^{1} \mathrm{C}$ Willberg, ${ }^{2} \mathrm{~B}$ Seigal, ${ }^{1} Y$ uHoi Kang, ${ }^{3} \mathrm{~J}$ Phillips-Hughes, ${ }^{4} \mathrm{~J}$ Collier, ${ }^{1} \mathrm{E}$ Barnes, ${ }^{2} \mathrm{R}$ Thimme, ${ }^{2} \mathrm{P}$ Klenerman. ${ }^{1}$ Peter Medawar Building for Pathogen Research, Oxford University; ${ }^{2}$ Department of Medicine, University of Freiburg; ${ }^{3}$ Radiology Department, Churchill Hospital, Oxford; ${ }^{4}$ Department of Gastroenterology, John Radcliffe Hospital, Oxford

Introduction $\gamma-\delta$ T cells have been found in blood/liver of patients infected with hepatitis C (HCV). CD161+ is a C-type lectin found on the surface of T cells in humans. HCV specific T-cells have been found to express CD161 with enrichment within the liver. The role \& function of CD161 $+\gamma-\delta$ cells has not been established in HCV. Aim To explore the phenotype and function of CD161+ $\gamma-\delta$ T cells in humans, and to assess the impact on these cells of HCV infection. Method Whole blood/Peripheral Blood Mononuclear cell (PBMC) antibody staining with subsequent flow cytometry was performed to assess phenotype. Function was examined by Intracellular Cytokine staining (ICS). Intrahepatic lymhocytes (IHLs) were isolated from patients having liver biopsies for clinical indications with paired blood samples. Cord blood samples were used after ethical approval gained and consent from mothers.

Results In healthy controls, the CD161 subset encompassed a mean of $82.1( \pm 12.8) \%$ of $\gamma-\delta$ cells. The CD161 subset expressed more CXCR3/CCR6/IL-18R ( $p=0.03 / 0.01$ and 0.03 respectively) than the CD161- subset. The CD161 + cells expressed significantly more If-y/ TNF- $\alpha$ ( $p=0.001$ respectively) than the negative subset, and more Granzyme A, B and K and perforin in keeping with a Th1 profile. $20 \%$ of $\gamma-\delta$ cells from cord blood samples expressed CD161, suggesting it is an innate feature but expanded throughout life. Whole blood $\gamma-\delta$ populations were significantly reduced in HCV compared to healthy donors $(70 \% \vee 40 \%, p=0.0031)$, with the proportion of CD161 $+\gamma-\delta$ T-cells reduced in chronic HCV $(82.1 \%$ vs $39 \%, p=0.006)$. The $\gamma-\delta$ cells in HCV expressed significantly more activation markers (CD38/CD69) and CXCR6 than in healthy controls irrespective of CD161 status. Of the CD161+ $\gamma-\delta$ cells, the predominant subset were $\mathrm{V} \delta 2$ in healthy controls blood however in HCV this was significantly reduced $(p=0.0173)$ with the V $\delta 1$ subset dominating. On isolation of IHLs $(n=17: 8 \mathrm{HCV}, 3 \mathrm{HBV}$ and rest NAFLD/NASH), there was an enrichment of CD3 $+\gamma-\delta$ T-cells in liver tissue compared to blood irrespective of CD161 status. On subanalysis there were no statistical differences between CD161 status of the $\gamma-\delta$ cell T-cells when comparing viral v non-viral aetiologies. Conclusion Our data suggests that HCV infection does directly reduce the $\gamma-\delta$ T-cell population in peripheral blood: mainly the CD161 subset. The virus also causes a reversal in the CD161 $\gamma-\delta$ T-cell population from $\mathrm{V} \delta 2$ to $\mathrm{V} \delta 1$ in the periphery, however it appears the enrichment of $\gamma-\delta \mathrm{T}$ cells to the liver is not specific to CD161 + cells or to HCV infection. The CD161 phenotype present at birth but expanded throughout life. The CD161 subset appears to display a more Th1 profile and unlike their CD8 CD161++ $\alpha-\beta$ counterparts, CD161 expression by $\gamma-\delta$ T-cells is not tightly linked to a Type-17 differentiation pathway.

\section{P93 NFKB ACTIVATION BY TLR AGONISTS IS DEFICIENT IN MACROPHAGES LACKING STAT2}

doi:10.1136/gutjnl-2011-300857a.93

${ }^{1} \mathrm{~W}$ Alazawi, ${ }^{2} \mathrm{~A}$ Gamero, ${ }^{1} \mathrm{H}$ Heath, ${ }^{3} \mathrm{P}$ Smith, ${ }^{4} \mathrm{M}$ Jacobs, ${ }^{1} \mathrm{~J}$ Waters, ${ }^{1} \mathrm{G}$ R Foster ${ }^{1}$ Barts \& The London School of Medicine; ${ }^{2}$ Temple University, Philadelphia, USA; ${ }^{3}$ St George's University London; ${ }^{4}$ University College London

Introduction Engagement of Toll-like receptors (TLRs) is an important initiator of the innate immune response in inflammatory liver diseases and leads to release of a range of inflammatory cytokines. TLRs signal through divergent signal transduction pathways but the majority lead to activation of members of the NFKB family. STAT2 is an essential component of canonical signalling through the type I and type III interferon (IFN) receptor but, unlike other members of the Stat family, is not believed to be involved in other signal transduction pathways.

Aim We examined the role of STAT2 on inflammatory signalling.

Results Loss of STAT2 markedly reduced immortalised macrophages' response to a range of TLR agonists (lipopolysaccharide (LPS), poly I:C and IL-1)-production of TNF $\alpha$ and RANTES proteins and a range of inflammatory mRNAs were decreased. The reduction in inflammatory cytokine production could be reversed by reconstitution with STAT2 but blockade of type I IFN signalling did not reproduce the phenotype. Restoration of the normal response to LPS could be achieved with tyrosine phosphorylation defective STAT2 indicating that STAT2 interacts with these signalling pathways without phosphorylation on Tyr690. The multiplicity of STAT2's effect suggests a common defect to these signalling pathways. There were no abnormalities in the activation of early signalling components in the absence of STAT2, however, levels of phosphorylated $I \kappa \mathrm{B}$, although not total $I \kappa \mathrm{B}$, were reduced in STAT2 $^{-/-}$cells. Phosphorylation of this inhibitor of NFKB leads to its degradation and release of NFKB proteins into the nucleus. We found that translocation of the NFKB protein p 65 into the nucleus and its subsequent binding to DNA was impaired in STAT2 deficient cells.

Conclusion These data suggest that STAT2 is a critical component of the TLR signalling response to early inflammatory stimuli, in particular through maintaining the normal phosphorylation of $I \kappa \mathrm{B}$.

\section{P94 RAPAMYCIN HELPS MAINTAIN THE REGULATORY PHENOTYPE OF CYTOCHROME P450IID6-SPECIFIC TREG EXPANDED FROM PATIENTS WITH AUTOIMIMUNE HEPATITIS TYPE 2 BY REDUCING THE NUMBER OF IFN $\gamma^{+}$ CELLS}

doi:10.1136/gutjnl-2011-300857a.94

B Holder, C R Grant, Y Ma, G Mieli-Vergani, D Vergani, M S Longhi. Institute of Liver Studies, King's College London School of Medicine at King's College Hospital, London, UK

Introduction Control of $\mathrm{T}$ cell reactivity to cytochrome P450IID6 (CYP2D6) is key to immune-tolerance restoration in autoimmune hepatitis type 2 (AIH-2). $\mathrm{CD} 4{ }^{+} \mathrm{CD} 25^{+}$regulatory $\mathrm{T}$ cells (Treg) central to autoreactive $T$ cell regulation, are impaired in AIH-2. Cell therapy based on CYP2D6-specific Treg (CYP-Treg) could provide specific control over effectors of liver damage in AIH-2. We have generated CYP-Treg from AIH-2 patients and demonstrated that these cells exert greater suppression than polyclonal Treg. Whether CYP-Treg can undergo expansion maintaining their functional phenotype is untested.

Aim To assess CYP-Treg functional phenotype over 2-week expansion in AIH-2 patients. 
Methods 48 CYP-Treg cell lines were obtained from $12 \mathrm{AIH}-2$ patients positive for the predisposing HLA-DR7 and DR3 alleles; 36 Treg cell lines specific for a DR7 or DR3-restricted influenzahaemagglutinin (HA) peptide were generated from $9 \mathrm{DR}^{+}$or $\mathrm{DR}^{+}$ healthy subjects (HS) and used as controls. CYP- and HA-Treg were obtained after co-culture with peptide-pulsed semi-mature DCs. T-reg were expanded for 2 weeks in the presence of: (1) IL2 (300 U/ $\mathrm{ml}$ ); (2) IL2 + rapamycin (RP) to enhance Treg function; (3) IL2+IL6/ IL1b, cytokines mimicking the proinflammatory milieu of AIH-2. Treg phenotype was determined by flow cytometry; frequency of cytokine-producing cells by intracellular staining.

Results Before expansion, the frequency of $\mathrm{CD}_{127^{-}}$and $\mathrm{FOXP3}^{+}$ cells exceeded $80 \%$ in both CYP- and HA-Treg. Compared to HATreg, CYP-Treg contained higher numbers of IFNg (6.4 \pm 1 vs $3.6 \pm 1.2, \mathrm{p}=0.09)$, IL2 (9.5 \pm 2.6 vs $2.6 \pm 0.5, \mathrm{p}=0.02)$, IL17 (7.1 \pm 1 vs $3 \pm 1.2, \mathrm{p}=0.026)$, IL10 (9.1 \pm 2 vs $3.3 \pm 2.6, \mathrm{p}=0.03)$ and TGFb $(10.4 \pm 2$ vs 3.6 $\pm 0.7, p=0.001)$ producing cells. After expansion with IL2, CYP- and HA-Treg maintained a similarly high frequency of $\mathrm{FOXP3}^{+}$and $\mathrm{CD} 127^{-}$cells, while frequency of $\mathrm{IFNg}^{+}$cells increased markedly (CYP-Treg: from $6.5 \pm 1$ to $27 \pm 3, \mathrm{p}<0.0001$; HA-Treg: from $3.8 \pm 1.5$ to $11 \pm 1.3, p<0.01)$. Exposure to RP decreased the frequency of IFNg ${ }^{+}$cells by $36 \%(p=0.04)$ in HS and by $30 \%(p=0.15)$ in AIH2. Exposure of Treg to IL6/IL1b had no effect on their phenotype and cytokine production.

Conclusion Compared to HA-Treg, CYP-Treg contain higher numbers of cytokine-producing cells, possibly reflecting a higher activation state of their precursors. After expansion, antigen-specific Treg retain a classical T-reg phenotype (CD127- and FOXP3+) even upon exposure to pro-inflammatory stimuli, but contain a high proportion of $\mathrm{IFNg}^{+}$cells. Reduction of $\mathrm{IFNg}^{+}$cells in the presence of $\mathrm{RP}$ suggests a role for this drug in the expansion of antigen-specific T-reg for immunotherapy in AIH-2.

\section{P95 MOLECULAR MECHANISMS OF MONOCYTE REPROGRAMMING IN ACUTE LIVER FAILURE: IMPORTANCE OF HEPATICALLY DERIVED ANTI-INFLAMMATORY MEDIATORS}

doi:10.1136/gutjnl-2011-300857a.95

${ }^{1} \mathrm{C} G$ Antoniades, ${ }^{2} \mathrm{~V}$ Zingarelli, ${ }^{3} \mathrm{~L}$ Possamai, ${ }^{2} \mathrm{R}$ Mistry, ${ }^{2} \mathrm{R} \mathrm{D}$ Abeles, ${ }^{2} \mathrm{M}$ Bruce, ${ }^{1} \mathrm{M} J$ W McPhail, ${ }^{2} \mathrm{~N}$ Heaton, ${ }^{2} \mathrm{G}$ Auzinger, ${ }^{2} \mathrm{~W}$ Bernal, ${ }^{2} \mathrm{D}$ Vergani, ${ }^{3} \mathrm{M} \mathrm{R}$ Thursz, ${ }^{2} J$ A Wendon. 'Institute of Liver Studies, King's College Hospital, Denmark Hill, London \& Liver and Anti-viral Centre, St Mary's Hospital, Imperial College London; ${ }^{2}$ Institute of Liver Studies, King's College Hospital, Denmark Hill, London; ${ }^{3}$ Liver \& Anti-viral Centre, St Mary's Hospital, Imperial College London

Introduction Monocytes from patients with acetaminophen-induced acute liver failure (AALF) bear striking phenotypic and functional similarities with endotoxin tolerant (ET) monocytes and may account for the marked predisposition to sepsis and increased
NF-kBp65 expression:Controls vs ALF
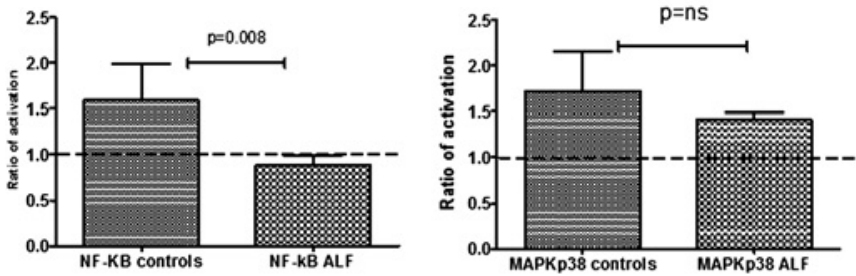

Abstract P95 Figure 1 Phosphoflow experiments investigating NFkBp65 and MAPKp38 expression following LPS challenge in study groups.

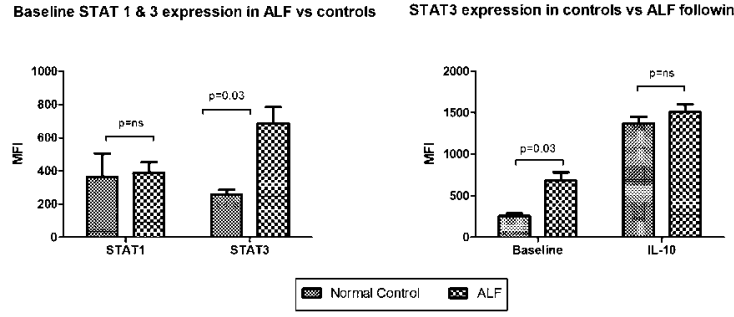

Abstract P95 Figure 2 Phosphoflow experiment investigating STAT-1 and STAT-3 expression in study groups at baseline and following exogenous IL-10 administration.

mortality in AALF. ET reprograms monocyte responses in response to lipolysaccharide (LPS) stimulation by reducing expression of proinflammatory (eg, TNF- $\alpha$, IL- 6 ) and augmenting the production of anti-inflammatory cytokines (eg, IL-10). At a molecular level, reductions in positive regulators (eg, NF-kBp65) of toll-like receptor (TLR)-4 dependent signalling pathway typify ET. Soluble antiinflammatory mediators, such as IL-10 and SLPI, exert negative regulation of TLR responses via STAT3 and NF-kBp65 dependent signalling pathways respectively.

Aim To delineate the molecular mechanisms and functional consequences of ET in AALF.

Method Following TLR-4 (LPS; $100 \mathrm{ng} / \mathrm{ml}), \operatorname{IFN}-\gamma(10 \mathrm{ng} / \mathrm{ml})$ and IL-10 $(50 \mathrm{ng} / \mathrm{ml})$ stimulation, phosphoflow technique was used to identify changes in regulators of TLR (NF-kBp65, MAPK p38), STAT-1 and STAT-3 signalling pathways in ex-vivo CD14+/CD33+ monocytes in eight AALF patients and 10 healthy volunteers (HC). Results expressed as MFI and ratio of activation (MFI following LPS/IFN- $\gamma /$ IL-10 stimulation divided by baseline MFI [RPMI]).

Serum TNF- $\alpha$, IL-10 and SLPI were measured by ELISA $(\mathrm{pg} / \mathrm{ml})$ in 34 AALF patients and 15 healthy volunteers (HC). Regional levels of TNF- $\alpha$, IL-10 and SLPI (portal vein [PV]), hepatic vein [HV]) were determined using in a further five AALF patients at time of liver transplantation (LT). Hepatic expression of TNF- $\alpha$, IL-10 and SLPI (all $\mathrm{pg} / \mathrm{ml}$ below) was determined using whole liver tissue homogenates from seven AALF explants and eight controls. Ex-vivo monocyte phagocytosis of FITC-labelled Escherichia coli was determined in five AALF and 10 healthy volunteers (HC) using FACS analysis.

Results In contrast to HC, TLR-4 stimulation markedly reduced NFkBp65 expression, while MAPKp38 signal transduction responses remained similar to that of $\mathrm{HC}$ (Abstract P95 figures 1-2). Baseline STAT-3 expression was significantly elevated in AALF patients compared to HC whereas no differences in STAT-1 expression was detected (Abstract P95 figure 2). Increase in STAT-3 expression following IL-10 stimulation was similar between AALF patients and HC.

AALF patients had significantly higher serum concentrations of TNF- $\alpha$ (21 vs $1.5 ; p<0.001)$, IL-10 (170 vs 40; $p<0.001)$ and SLPI (71 200 vs $43310 ; \mathrm{p}<0.001)$ compared to HC. A trans-hepatic (HV > PV) gradient was seen for IL-10 and SLPI but not for TNF- $\alpha$ in 4 out

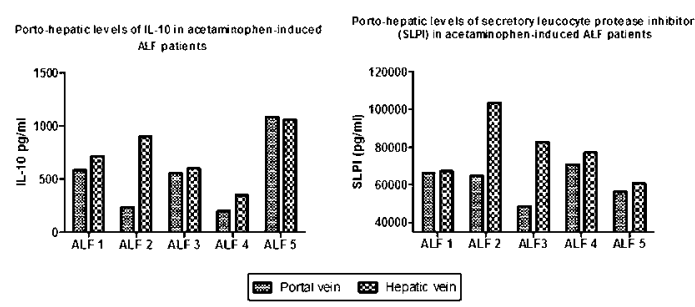

Abstract P95 Figure 3 Regional levels of SLPI and IL-10 in five patients with AALF. 